\title{
Histometry of normal thyroid in man
}

\author{
RA BROWN,* M AL-MOUSSA, $\dagger$ J SWANSON BECK $\dagger$ \\ From the Departments of *Mathematics and $\dagger$ Pathology, University of Dundee, Ninewells Hospital and \\ Medical School, Dundee
}

SUMMARY Thyroids that did not show any histopathological evidence of disease were collected at necropsy from 107 patients who did not have any history of endocrine disease. Thyroid volume increases with age during childhood and adolescence, remains stable in younger adults, and declines in older subjects. There was no simple mathematical function relating thyroid volume with age: robust smooth curve fitting, however, permitted objective assessment of the age changes. The volume proportion $\left(\mathrm{V}_{\mathrm{v}}\right)$ measurements of the various histological components did not show any important change with age, but the measurements of volume of these components reflected the gland volume changes with age. The total number of acini in the gland did not show any great age changes, and so it was concluded that the volume changes were a consequence of changes in the size of the individual acini. Measurements of internal and external surface area of the acini showed an increase in childhood and adolescence and decline in older age: unlike the other measurements reported in this paper, however, the surface area measurements must be considered as relative because of the Mandelbrot effect. The prevalence of microscopic nodules in otherwise normal thyroids increased in older patients.

There are numerous descriptions of the microscopic appearance of diseased and healthy thyroids. Early attempts were made to count the number of acini ${ }^{1}$ and to measure the height of the epithelial cells by direct measurement, but progress toward meaningful measurements ${ }^{2}$ became possible only with the application of various sampling and measuring techniques based on stereological principles. ${ }^{4}$ In the technical sense the human thyroid has proved to be a particularly suitable subject for histometric study because the elements are easy to identify and clearly delineated; furthermore, the constituent units (the acini) do not show any consistent spatial orientation-that is, the tissue can be considered, for practical purposes, to be isotropic.

This paper describes measurements in a series of patients in whom the thyroid can reasonably be regarded as "normal" as the patients did not have any clinical history of endocrine disease, and no histological abnormality was noted by an experienced observer. Certain of the categories of measurement recorded in this study have been previously reported by Roberts: ${ }^{3}$ in general, we confirm his results, but we differ substantially in measurements of the internal

Accepted for publication 16 January 1986 surface area of acini. We also report measurements that have not been recorded previously-namely, the external surface area of the acini and estimates of the total number of acini in the gland.

\section{Material and methods}

The thyroid was taken at necropsy from 107 patients, aged between 1 day and 93 years; patients selected for this study did not have any clinical history of endocrine disease, and no recognisable histopathological changes were seen in the endocrine glands. Forty six patients had died suddenly outside hospital (mainly from accidents): the remainder of the glands were taken from patients dying in Ninewells Hospital, Dundee. Body surface area was calculated from height and weight. ${ }^{5}$

\section{HISTOMETRIC METHODS}

After dissection to remove extraneous tissue the volume of the fresh gland was measured by the fluid displacement method. ${ }^{6}$ The bath was filled with $4 \%$ neutral buffered formaldehyde, and the thyroid gently placed in the fluid, and when necessary, light pressure with the tip of a mounted needle was used to ensure complete immersion: the displaced fluid was collected in a measuring cylinder $(50 \mathrm{ml}$ capacity for a large 
Table 1 Estimate of relative standard error of counts of whole acinar unit (epithelial wall and contents) in 103 subjects (whole series except infants)

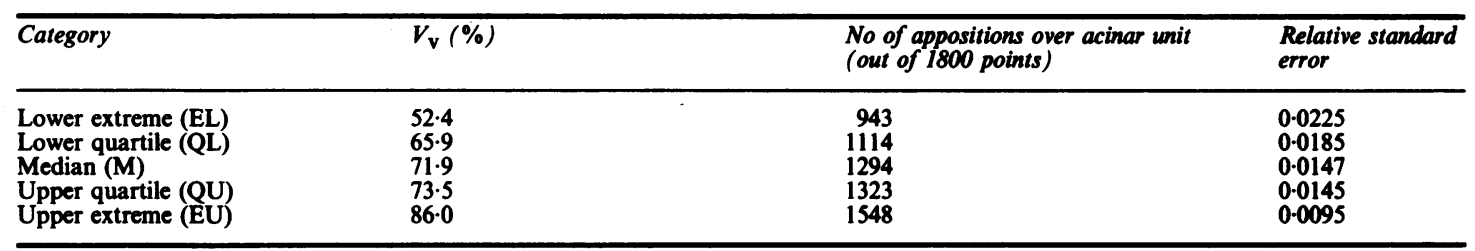

thyroid and $25 \mathrm{ml}$ for a smaller gland). This procedure was performed in triplicate, particular care being taken to ensure that there was no carry over of fluid on the surface of the gland or inside the measuring cylinder. The mean value was used for subsequent calculations.

The gland was fixed whole in $4 \%$ neutral buffered formaldehyde for 24 to 48 hours, and then cut in the horizontal plane into $10 \mathrm{~mm}$ thick slices to permit complete fixation with minimal twisting. The portions of tissue were recut in the transverse plane into $5 \mathrm{~mm}$ thick slices and fixation continued for a further 24 to 48 hours, and then the volume of these pieces of tissue was remeasured by the fluid displacement method. At least five blocks were selected from scattered positions in the gland, and the linear dimensions of the face were recorded. The blocks were trimmed to $2 \mathrm{~mm}$ thickness before processing to paraffin with standard methods; $5 \mu \mathrm{m}$ sections were stained with Mayer's haemalum and eosin, and the linear dimensions of the stained section of the face were measured. The extent of shrinkage was measured at two stages ${ }^{7}$ : by comparing the volume before and after fixation the correction factor $\mathrm{f}^{3}$ for primary fixation could be determined; and by comparing the linear dimensions before and after processing, the linear shrinkage factor $p$ (calculated as the mean values for the five sections from each gland) was determined and the corresponding volume shrinkage factor $\mathrm{p}^{3}$ calculated. ${ }^{78}$ The overall linear shrinkage factor from fresh tissue to stained section, $F$, was the product of these two indices, $f$ and $p$, and the corresponding $F^{2}$ and $F^{3}$ values were used to correct area and volume histometric measurements.

The primary histometric measurement was the determination of the volume proportions $\left(V_{v}\right)$ of the histological components of the gland. The point counting method was applied to all sections using a 36 point square eyepiece graticule (Leitz No 519902) in the stratified random sampling approach. ${ }^{9}$

Each slide was positioned on the microscope stage so that the objective overlay a corner of the section before microscopy was started. After the first field had been counted the slide was moved under stage micrometer control in increments of $1 \mathrm{~mm}$ trans- versely and vertically until 10 fields were counted in each section. Examination of five sections therefore yielded 1800 points for each gland. The acinar epithelium, colloid, stroma, blood vessels, and lymphoid tissue were readily identified: the retraction space occasionally present between the epithelium and the outer margin of the colloid was classified separately. The convention used for decision when a point overlay a boundary was that this was attributable to the adjacent structure above and to the left. The estimate of the acinar unit was calculated by totalling the point counts for epithelium, colloid, and retraction space: that for the acinar lumen was obtained by the addition of points over colloid and retraction space. The main purpose of this investigation was to determine the range of variation in the size of the acinar units in endocrinologically normal people in Tayside, and so the precision of the measurements of these features was studied in detail for all subjects (except infants). Table 1 shows that even for the lowest value in the series, the relative standard error (RSE) for the measurement $V_{v}$ for the acinar unit was $2 \cdot 25 \%$. The estimates for the other components were necessarily less precise, but these were generally less than $10 \%$ for measurements of the epithelial content of the gland. All $\mathrm{V}_{\mathrm{y}}$ data were corrected to absolute values by multiplying by the shrinkage factor $\left(\mathrm{F}^{3}\right)$.

The number of acini in the thyroid was calculated by the modified Weibel and Gomez ${ }^{10}$ method using the formula:

$$
N_{V}=\frac{K_{A}^{1.5}}{\beta V_{V}^{0.5}}:\left(N=N_{V} V_{\text {acinar unit }}\right)
$$

The acinus in the thyroid is not strictly spherical but approximates in shape to a dodecahedron because of close packing, and so $\beta$ was given the value $1.55 .^{11}$ The constant $K$ was introduced into the equation ${ }^{12}$ to take account of the variation in size of the objects being counted and is proportional to the ratio of the third to the first moments of the size distribution ${ }^{13}$ : by modelling this size distribution as a gamma distribution, which can be made to approximate closely to different extents of spread and skewing by modification of the value attributed to " $n$ ", Weibel 11 showed that for particle size distributions with a 
coefficient of variation of less than $20 \% \mathrm{~K}$ will take a value between 1.0 and 1.05; and so its introduction into the calculation of $\mathrm{N}_{\mathbf{v}}$ would modify the result by no more than 5\%: he concluded that $\mathrm{K}$ can be neglected for many biological applications. It was impossible to measure the size distribution of acini in this investigation, and so it was decided to allocate $\mathbf{K}$ the arbitary value of 1.0 (knowing that this approximation would not introduce gross errors) to avoid unwarranted assumptions on the extent of variation and skewing of the acinar size distribution. $\mathbf{N}_{\mathbf{A}}$ was measured in 20 fields on each of the five sections from each gland with a square graticule at magnification $\times 120$, with the convention that acini transected by the upper and left margins were accepted and that those crossing the other borders were disregarded. In the calculations of $N_{v}$ the values for $N_{A}$ and $V_{V}$ were corrected for shrinkage by $F^{2}$ and $F^{3}$ respectively.

The surface area was measured initially on the basis of the relation ${ }^{6}$ :

$$
S_{\mathbf{V}}=2 I_{L}: S=S_{V} V_{\text {acinar unit }}
$$

The mean linear intercept $I_{L}$ was measured separately for the internal and external surfaces of the acini in $\mathbf{2 0}$ fields in the sections from the five blocks of each thyroid at a magnification of $\times \mathbf{4 0 0}$ with a cross hair graticule (Leitz No 519904): $F$ was applied as shrinkage factor as this datum was linear. When it was realised that the calculated size of the internal surface area differed by an order from the only previous report, ${ }^{3}$ it was decided to compare our linear intercept results with measurements obtained by a less rigorous method based on different stereological assumptions, previously used to measure the surface area of the alveoli of the lung. ${ }^{14}$ This depends on the relation:

total acinar surface area $=$ No of acini $\times$ estimate of mean surface area of acini

or,

$$
S_{\text {acini }}=N_{\text {acini }}\left[\frac{V_{\text {acini }}}{N_{\text {acini }}}\right]^{0.67} \sigma
$$

where $\sigma$ is a shape factor $\left(4.8\right.$ for spheres $\left.{ }^{14}\right)$. These second calculations were performed on the shrinkage corrected values for $\mathbf{N}$ and $\mathbf{V}$ for the acinar unit and acinar lumen, respectively, in calculations of the external and internal surface areas.

\section{STATISTICAL METHODS}

Preliminary study of the histometric findings showed evidence of changes with age and the existence of differential population variability with age. To facilitate subsequent statistical analysis we attempted to make the extent of variability more uniform with age by logarithmic, square root, and cube root transformation, but none of these simple transformations

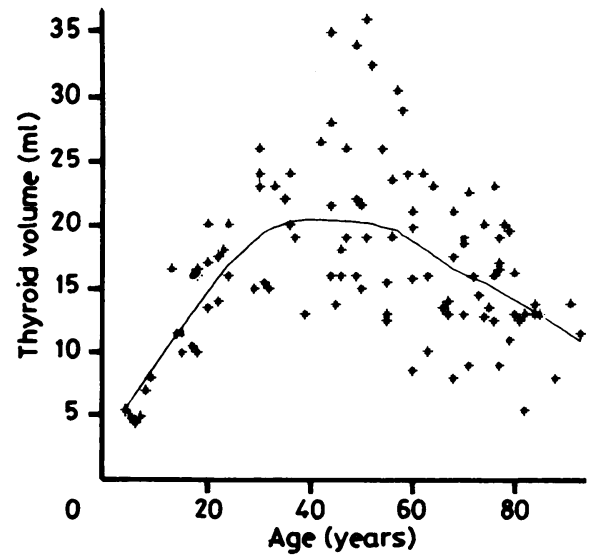

Fig. 1 Variation of thyroid volume with age. Solid line indicates robust smooth curve fitted to data in this and all subsequent diagrams.

reduced the dependence of variability with age. Accordingly, "robust smooth curves" were fitted to the data using the method of Cleveland ${ }^{15}$ as this approach does not make any prior assumption on the distribution and variability of the data: the advantage of this method is that the resultant curve is not influenced by outliers, differential variability, or nonnormality of the data and it is calculated in an objective manner; the disadvantage is that the curve cannot be expressed by a simple mathematical function.

The technique of point counting provides information on the proportion of a tissue formed of the various histological components: variation of such data will show a dependence on mean. Accordingly, the arcsin transformation ${ }^{16}$ was made to validate subsequent statistical analysis.

The findings on the histometric measurements and calculated values for the various histological features of the thyroids of men and women showed overlapping distributions without evidence of systematic sex bias: the results were therefore pooled before analysis.

\section{Results}

Fig. 1 shows the measurements of thyroid volume and Table 2 summarises the extent of variation at different ages. The volumes of the glands of men and women did not seem to be differently distributed. The robust smooth curve showed that thyroid volume increased with age during childhood and adolescence, remained fairly constant in younger adults, and declined more slowly in older people. The variability increased progressively as the thyroid grew in volume in the younger group, but it remained relatively unchanged 
Table 2 Summary of histometric findings in thyroids

\begin{tabular}{|c|c|c|c|c|c|c|c|c|c|c|c|}
\hline \multirow{2}{*}{$\begin{array}{l}\text { Decade, No of } \\
\text { subjects, and } \\
\text { measurement } \\
\text { category }\end{array}$} & \multirow{2}{*}{$\begin{array}{l}\text { Total thyroid } \\
\text { volume }(\mathrm{ml})\end{array}$} & \multicolumn{2}{|c|}{$V_{\mathrm{v}}$ Epithelium } & \multicolumn{2}{|c|}{$\begin{array}{l}V_{\mathrm{v}} A c i n a r \\
\text { lumen }\end{array}$} & \multicolumn{2}{|c|}{$V_{\mathbf{v}}$ Stroma } & \multicolumn{2}{|c|}{$V_{\mathbf{v}}$ Vessels } & \multicolumn{2}{|c|}{$V_{\mathrm{v}}$ Lymphos } \\
\hline & & $(\%)$ & $m l$ & $(\%)$ & $m l$ & $(\%)$ & $m l$ & $(\%)$ & $m l$ & $(\%)$ & \\
\hline 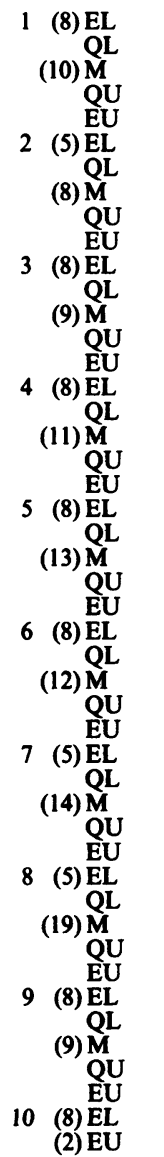 & 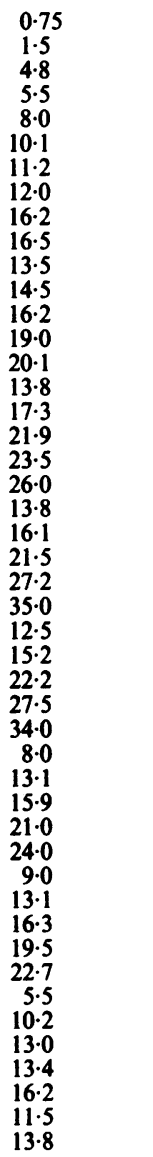 & $\begin{array}{r}13 \cdot 3 \\
13 \cdot 9 \\
19 \cdot 8 \\
24 \cdot 7 \\
31 \cdot 1 \\
10 \cdot 3 \\
11 \cdot 4 \\
13 \cdot 1 \\
15 \cdot 3 \\
20 \cdot 3 \\
10 \cdot 0 \\
11 \cdot 1 \\
11 \cdot 4 \\
13 \cdot 8 \\
18 \cdot 6 \\
8 \cdot 6 \\
11 \cdot 1 \\
11 \cdot 7 \\
12 \cdot 7 \\
14 \cdot 6 \\
8 \cdot 0 \\
20 \cdot 7 \\
21 \cdot 9 \\
23 \cdot 2 \\
27 \cdot 0 \\
11 \cdot 1 \\
12 \cdot 7 \\
14 \cdot 9 \\
17 \cdot 7 \\
22 \cdot 8 \\
9 \cdot 2 \\
11 \cdot 5 \\
12 \cdot 2 \\
13 \cdot 7 \\
17 \cdot 2 \\
11 \cdot 1 \\
11 \cdot 5 \\
13 \cdot 0 \\
16 \cdot 5 \\
18 \cdot 0 \\
11 \cdot 3 \\
12 \cdot 0 \\
13 \cdot 0 \\
14 \cdot 0 \\
22 \cdot 0 \\
16 \cdot 3 \\
17 \cdot 0\end{array}$ & 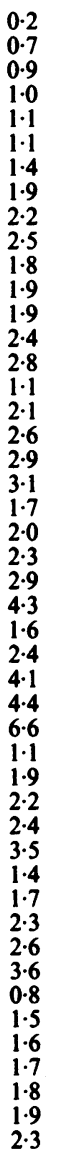 & $\begin{array}{l}13 \cdot 8 \\
17 \cdot 9 \\
43 \cdot 5 \\
47 \cdot 3 \\
57 \cdot 9 \\
46 \cdot 6 \\
51 \cdot 9 \\
57 \cdot 5 \\
59 \cdot 4 \\
66 \cdot 3 \\
48 \cdot 2 \\
54 \cdot 6 \\
61 \cdot 3 \\
63 \cdot 7 \\
68 \cdot 4 \\
50 \cdot 0 \\
58 \cdot 1 \\
59 \cdot 4 \\
63 \cdot 7 \\
67 \cdot 7 \\
39 \cdot 0 \\
53 \cdot 0 \\
56 \cdot 0 \\
60 \cdot 8 \\
64 \cdot 9 \\
41 \cdot 0 \\
46 \cdot 3 \\
52 \cdot 5 \\
60 \cdot 0 \\
63 \cdot 7 \\
41 \cdot 0 \\
47 \cdot 2 \\
53 \cdot 0 \\
61 \cdot 8 \\
66 \cdot 4 \\
45 \cdot 0 \\
52 \cdot 5 \\
57 \cdot 1 \\
65 \cdot 7 \\
74 \cdot 4 \\
43 \cdot 0 \\
45 \cdot 2 \\
56 \cdot 1 \\
57 \cdot 9 \\
59 \cdot 0 \\
51 \cdot 0 \\
59 \cdot 1\end{array}$ & $\begin{array}{r}0.1 \\
0.6 \\
2.0 \\
3.4 \\
4.2 \\
5.0 \\
5.9 \\
8.2 \\
9.6 \\
10.6 \\
6.7 \\
8.2 \\
10.5 \\
12.2 \\
12.8 \\
8.8 \\
9.9 \\
11.6 \\
14.2 \\
16.8 \\
7.3 \\
8.9 \\
11.9 \\
15.6 \\
19.8 \\
5.0 \\
8.6 \\
10.8 \\
13.4 \\
22.9 \\
3.3 \\
6.7 \\
8.4 \\
10.5 \\
16.0 \\
4.5 \\
7.3 \\
9.5 \\
11.7 \\
13.6 \\
3.1 \\
4.5 \\
7.3 \\
7.8 \\
8.7 \\
6.8 \\
7.0\end{array}$ & $\begin{array}{l}23 \cdot 5 \\
28 \cdot 2 \\
34 \cdot 7 \\
41 \cdot 4 \\
52 \cdot 9 \\
18 \cdot 3 \\
22 \cdot 2 \\
24 \cdot 2 \\
26 \cdot 3 \\
30 \cdot 1 \\
18 \cdot 5 \\
22 \cdot 2 \\
24 \cdot 4 \\
26 \cdot 5 \\
28 \cdot 9 \\
8 \cdot 2 \\
19 \cdot 6 \\
22 \cdot 7 \\
25 \cdot 4 \\
48 \cdot 2 \\
20 \cdot 0 \\
23 \cdot 1 \\
26 \cdot 1 \\
33 \cdot 1 \\
43 \cdot 0 \\
20 \cdot 9 \\
23 \cdot 2 \\
26 \cdot 1 \\
27 \cdot 9 \\
34 \cdot 8 \\
16 \cdot 6 \\
21 \cdot 7 \\
28 \cdot 7 \\
33 \cdot 2 \\
37 \cdot 1 \\
11 \cdot 2 \\
19 \cdot 0 \\
23 \cdot 8 \\
29 \cdot 4 \\
31 \cdot 7 \\
24 \cdot 0 \\
24 \cdot 7 \\
28 \cdot 0 \\
33 \cdot 8 \\
36 \cdot 0 \\
22 \cdot 0 \\
29 \cdot 0\end{array}$ & 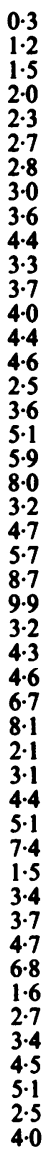 & 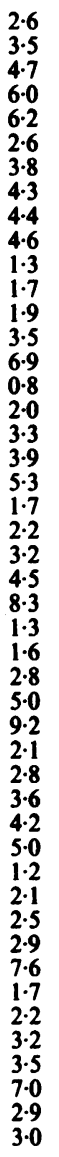 & $\begin{array}{l}0.1 \\
0.1 \\
0.2 \\
0.2 \\
0.4 \\
0.4 \\
0.5 \\
0.5 \\
0.6 \\
0.8 \\
0.2 \\
0.3 \\
0.4 \\
0.5 \\
1.0 \\
0.3 \\
0.3 \\
0.6 \\
0.9 \\
1.2 \\
0.4 \\
0.4 \\
0.5 \\
1.2 \\
1.5 \\
0.2 \\
0.3 \\
0.6 \\
1.1 \\
2.4 \\
0.2 \\
0.3 \\
0.6 \\
0.7 \\
1.0 \\
0.2 \\
0.3 \\
0.4 \\
0.5 \\
1.8 \\
0.1 \\
0.3 \\
0.4 \\
0.5 \\
0.6 \\
0.4 \\
0.4\end{array}$ & $\begin{array}{l}0.0 \\
1.0 \\
2.2 \\
3.0 \\
3.5 \\
0.3 \\
0.7 \\
1.3 \\
2.1 \\
2.9 \\
0.6 \\
0.7 \\
1.1 \\
1.3 \\
2.5 \\
0.1 \\
0.6 \\
1.1 \\
1.5 \\
2.4 \\
0 \\
0.8 \\
1.1 \\
1.6 \\
2.1 \\
0 \\
0.5 \\
0.7 \\
1.5 \\
5.8 \\
0.7 \\
1.1 \\
1.4 \\
1.9 \\
3.3 \\
0.6 \\
1.0 \\
1.4 \\
1.9 \\
3.0 \\
0.8 \\
1.3 \\
1.4 \\
2.1 \\
3.5 \\
0.1 \\
0.7\end{array}$ & 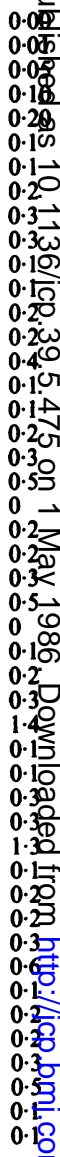 \\
\hline
\end{tabular}

in the older subjects. As a simple mathematical function could not be fitted to the data it was arbitrarily separated into three age groups: for those less than 20 years, the regression line of thyroid volume with age showed a positive slope that was significant at the $0.1 \%$ level, indicating postnatal growth; in the middle group (20-50 years) the regression line was not significant, indicating a constancy of volume in this range; and in the older patients ( $>50$ years) the regression slope was negative and significant at the $0 \cdot 1 \%$ level, indicating a slow progressive atrophy with increasing age.

\section{MEASUREMENT OF CONTENT OF VARIOUS}

HISTOLOGICAL COMPONENTS IN THE THYROID

Table 2 summarises the results of the point counting studies. The findings on $\mathbf{V}_{\mathbf{V} \text { acinar unit }}$ are shown in Figs. 2 (original data) and 3 (arcsin transformed data). The robust smooth curve fitted to the data suggested very little change with age. Linear regression analysis of the arcsin transformed data showed that the slope of the regression line was not significantly different from zero, confirming the absence of age dependence in $\mathbf{V}_{\mathbf{V}}$ acinar unit. Essentially similar results were seen in plots and statistical analysis of the components of the acinus (epithelium and lumen) and of the interstitial tissue.

The absolute volumes of the various histological components were calculated by multiplication of the thyroid volume by the appropriate $V_{v}$ value (expressed as a proportion). Fig. 4 shows the age correlation of the total acinar volume with age: the robust 


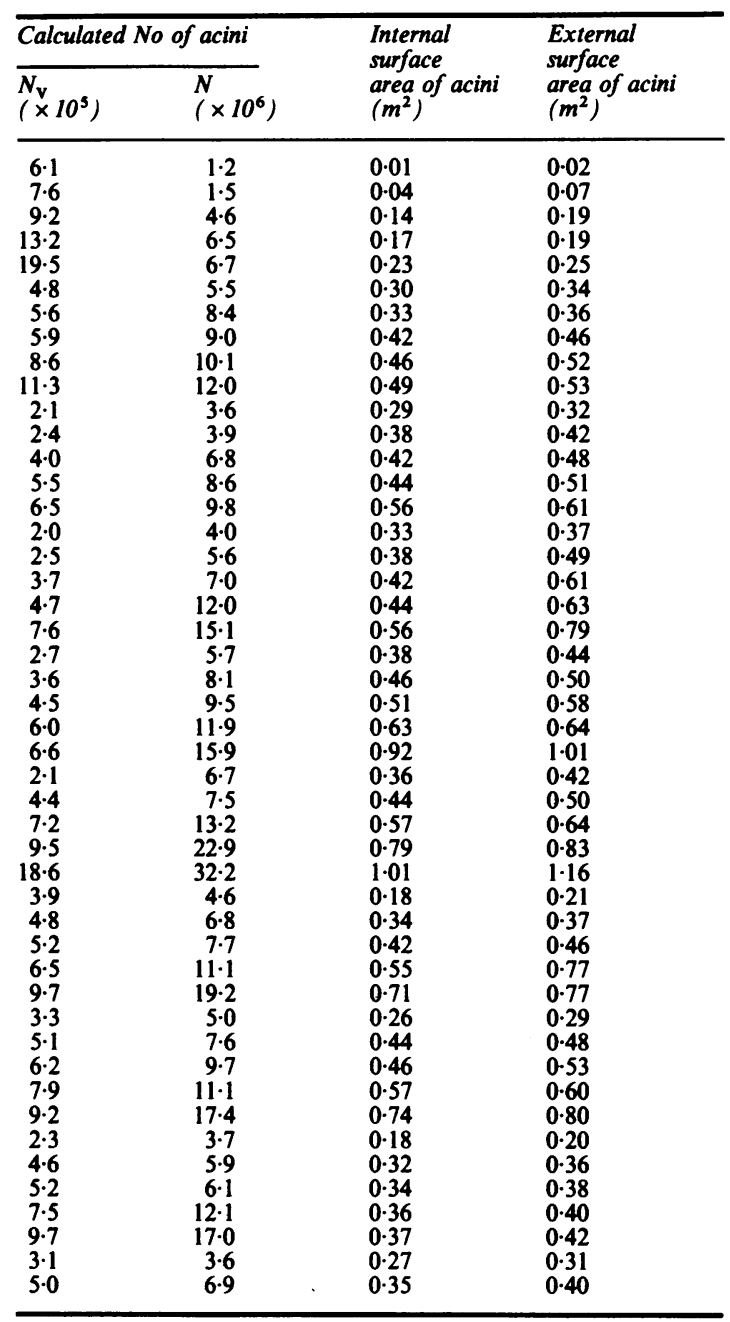

smooth curve fitted to these data was generally similar to that of the volume of the whole thyroid. Clearly, the derived values will have been powerfully influenced by the dependence of gland volume with age described above. The calculated values for the gland content of its various histological components (epithelium, lumen, and stroma) had age dependence similar to that of the whole acinar unit.

\section{Number of acini in the thyroid}

The scatter plot of $\mathbf{N}_{\mathbf{v}}$ for acini in glands showed considerable age variation (Fig. 5): the robust smooth curve indicated that $\mathbf{N}_{\mathbf{v}}$ falls in childhood and adolescence and shows a slower age related rise in older people. Log transformation of the data did not change

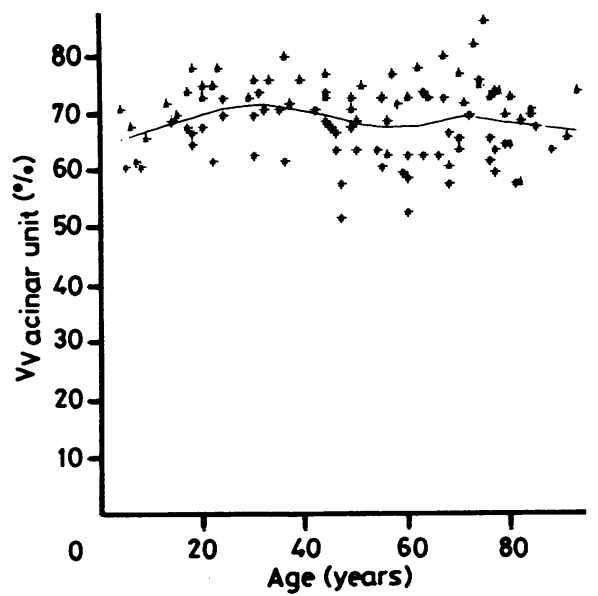

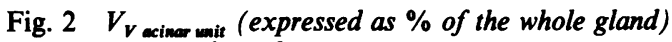
shows no strong dependence on age.

the downward trend in subjects less than 20 years old (Fig. 6): as selection of different values for $\mathrm{K}$ in the Weibel and Gomez equation will merely cause a rise or a fall in the level of the log plot without changing its shape, we deduced that our arbitrary decision to allocate a unitary value could not have concealed another age dependent relation of $\mathrm{N}_{\mathbf{v}}$.

The total number of acini (N) in the thyroid was calculated as the product of $\mathrm{N}_{\mathbf{v}}$ with $\mathrm{V}$. The robust smooth curve for $\mathrm{N}$ was relatively flat (Fig. 7), presumably because the corresponding curves for $N_{v}$ and $\mathrm{V}$ had complementary slopes in the younger and older subjects, and so the age effects tended to cancel each other out.

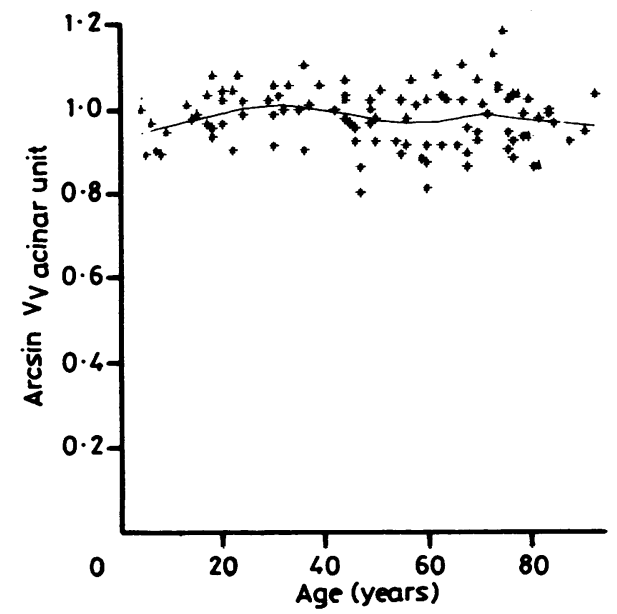

Fig. $3 V_{V a c i n a r}$ unit after arcsin transformation does not show dependence on age. 


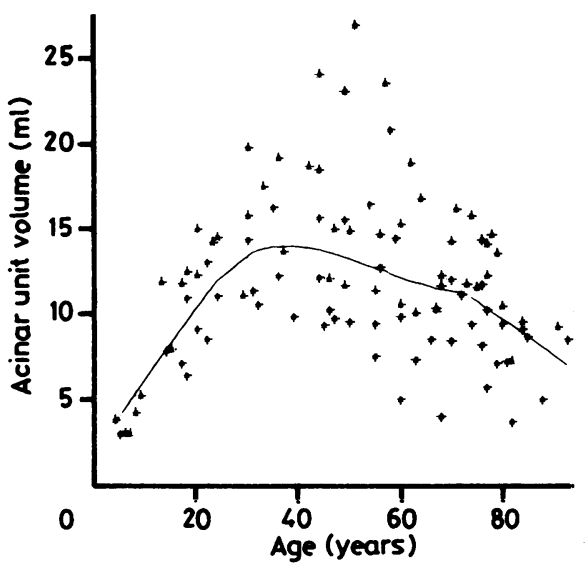

Fig. 4 Variation in calculated volume of acinar unit with age. Robust smooth curve fitted to these data is generally similar to that fitted to whole thyroid volume (Fig. 1).

\section{Surface area of acini}

The internal surface area of acini per unit volume of the gland was measured by the mean linear intercept method and showed very little apparent age dependent variability (Fig. 8). Linear regression analysis showed that the slope of the regression line was not significantly different from zero. The total internal surface area showed an age distribution analagous to that of the total thyroid volume (Fig. 9): this was not surprising as it was calculated by multiplication of the $S_{\mathbf{v}}$ internal surface area value by $\mathbf{V}$. When $S_{\text {internal surface area }}$ was calculated by the alternative geometric method in general, similar results were obtained. The measurements of $S_{\text {external surface area }}$ and $S_{\mathbf{v}}$ external surface area were proportionately larger than those of the internal surface and showed the age

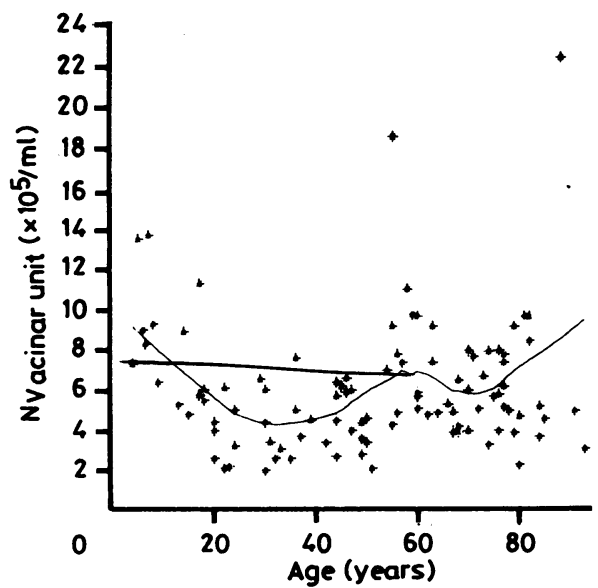

Fig. 5 Variation in $N_{\mathrm{v}}$ for acinar units with age.

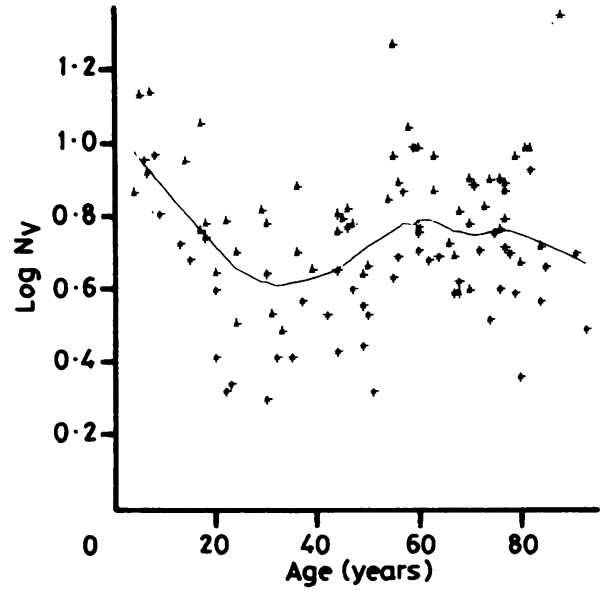

Fig. 6 Log transformed $N_{\mathrm{v}}$ data. This distribution shows persistence of downward trend in subjects less than 20 years old.

relations analagous to those of the internal surface.

Presence of microscopic nodules

Table 3 lists the number of microscopic nodules that were seen in sections of the five blocks from each thyroid. These nodules were not seen in children and young adults, and they became more common in older subjects. The nodules were excluded from the histometric measurements. No thyroid with macroscopic nodules was included in this survey.

\section{Discussion}

Visual inspection of the results of this histometric study showed that there were obvious age related changes in the thyroid. No simple mathematical func-

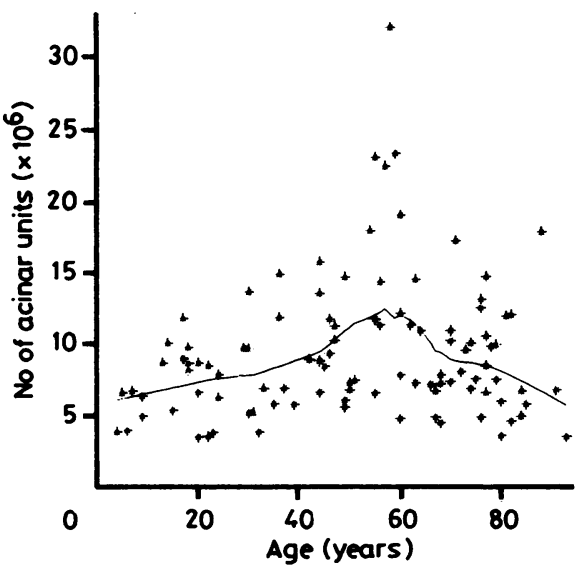

Fig. 7 Variation of $N$ (acinar unit) with age. 


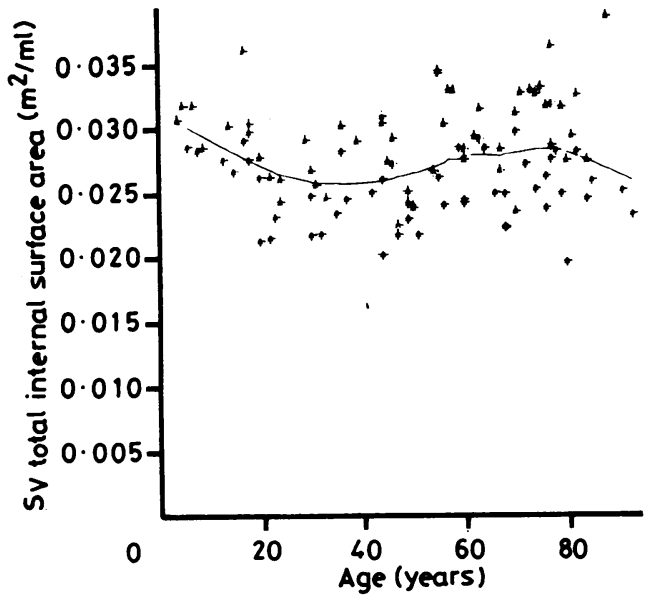

Fig. $8 S_{V \text { int }}$ shows no strong dependence on age.

tion was identified that explained the age variability, but robust smooth curve fitting permitted objective assessment of the results. It was clear that the proportion of the gland occupied by the various histological components did not change appreciably with age, but the total thyroid volume increased quite rapidly with postnatal growth and declined gradually in middle and old age, and that this factor dominated the derived measurements such as total acinar volume and surface area. There was less variability during postnatal growth, presumably because there was less scope in smaller glands, but it was also likely that there was less influence from acquired disease. The variability was much greater in the older subjects, but the cause was not identified. The calculated number of

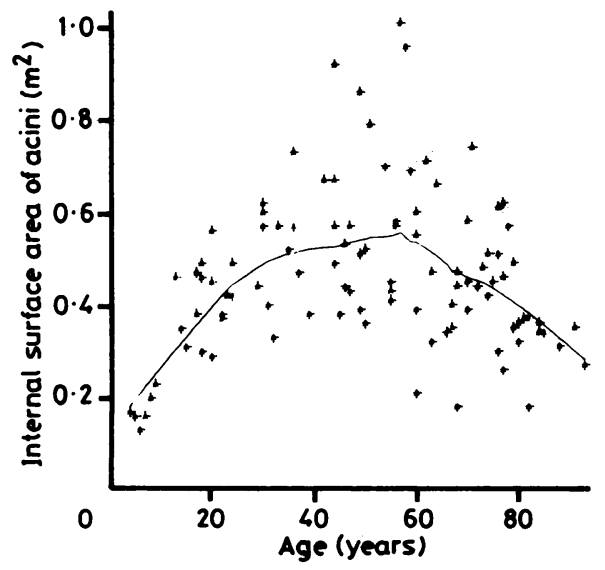

Fig. 9 Variation of $S_{i m t}$ with age. Robust smooth curve shows an analagous age dependence to curves shown in Figs. 1 and 4.
Table 3 Age incidence of microscopic nodules in thyroid

\begin{tabular}{lll}
\hline Decade & $\begin{array}{l}\text { No of thyroids } \\
\text { studied }\end{array}$ & $\begin{array}{l}\text { No of thyroids (each studied in five } \\
\text { histological sections) with } \\
\text { microscopic nodules }\end{array}$ \\
\hline First & 10 & 0 \\
Second & 8 & 0 \\
Third & 9 & 0 \\
Fourth & 11 & 2 \\
Fifth & 13 & 3 \\
Sixth & 12 & 2 \\
Seventh & 14 & 6 \\
Eighth & 19 & 4 \\
Ninth & 9 & 3 \\
\hline
\end{tabular}

acini in the thyroid did not seem to change greatly with age, and we concluded that the changes in total thyroid volume had resulted from enlargement of individual acini in childhood and adolescence and that the slower atrophy in old age arose from gradual shrinkage of acini: no attempt, however, was made to determine the extent to which these changes affected all acini, or were selective on a subpopulation. This study has shown that in subjects with no clinical evidence of thyroid disease the histological structure of the thyroid was relatively stable throughout life - that is, there seems to be a state of morphostasis in which structure is related to the functional demands of the gland.

The general distribution of the values for thyroid volume, epithelial volume, and acinar lumen in relation to age reported by Roberts $^{3}$ are similar to those presented in this paper. Our estimates for internal surface area of the acini, however, are ten times smaller: we consider that it is highly improbable that this large difference could be attributed to geographical differences in diet or environment, or to genetic factors. We believe that our results are a valid estimate (within the limits of the technique we used), because with prior knowledge of the reported values our measurements, calibrations, and calculations were checked and cross checked by ourselves and our colleagues. It is possible, however, that the higher values reported by Roberts could have been a consequence of measurement of the mean linear intercept at a higher magnification: the Mandelbrot effect ${ }^{17}$ will result in a larger estimate of the surface area, as increasing magnification will result in greater resolution. Unfortunately, Roberts ${ }^{3}$ did not record the conditions under which the measurements were made, but the use of projected images suggests a higher magnification.

Our study and that of Roberts ${ }^{3}$ have shown that it is practicable to make histometric measurements on the thyroid in man. This means that it should be possible to define the structural adaptations to physiological change, or disease, more precisely than has been possible to date. Absolute measurements of the 
volume of components and of numbers of acini is possible, but for studies of surface area care should be taken to include appropriate controls collected and measured under the same conditions as the study group, because such measurements can only be relative due to the Mandelbrot effect.

MAM was supported on a training fellowship from the government of the Republic of Syria. JSB was in receipt of a grant from the Scottish Hospital Endowments Research Trust (HERT 423).

Dr Rushton of the department of forensic medicine kindly supplied the thyroids from the patients who died suddenly outside hospital.

We are grateful to $\mathrm{Mr} R$ Fawkes and $\mathrm{Mr} \mathrm{S}$ MacPherson for the preparation of the diagrams and to the medical laboratory scientific officers of the department of pathology, Ninewells Hospital and Medical School, for excellent technical help in producing the numerous histological sections. We also thank Mrs Rosalind Mitchell for typing the manuscript.

\section{References}

${ }^{1}$ Jackson JL. The shape and size of the human thyroid follicle in health and disease. Anat $\operatorname{Rec} 1931 ; 48: 219-39$.

${ }^{2}$ Beck JS, Younge RJ, Simpson JG, et al. Lymphoid tissue in the thyroid gland and thymus of patients with primary thyrotoxicosis. Br J Surg 1973;60:769-71.

${ }^{3}$ Roberts PF. Variation in the morphometry of the normal human thyroid in growth and aging. $J$ Pathol 1974;112:161-8.
${ }^{4}$ Underwood EE. Quantitative stereology. Reading, Massachusetts Addison-Wesley, 1970.

${ }^{5}$ Bell GH, Davidson JN, Scarborough H. Textbook of physiology and biochemistry. 7th ed. Edinburgh: Churchill-Livingstone, 1968:216.

${ }^{6}$ Aherne WA, Dunnill MS. Morphometry. London: Edward Arnold, 1982:10.

7 Aherne WA, Dunnill MS. Morphometry. London: Edward Arnold, 1982:20.

${ }^{8}$ Weibel ER. Stereological methods. Vol 1. London: Academic Press, 1979:152.

${ }^{9}$ Younge RJ, Beck JS, Michie W. The predictive value of histometry of thyroid tissue in anticipating hypothyroidism after subtotal thyroidectomy for thyrotoxicosis. J Clin Pathol 1975; 28:94-8.

${ }^{10}$ Weibel ER, Gomez DM. Geometry and dimensions of the human airways. Fed Proc 1962;21:439.

11 Weibel ER. Stereological methods. Vol 1. London: Academic Press, 1979:44.

${ }^{12}$ Weibel ER, Kistler GS, Scherle WF. Practical stereological methods for morphological cytology. J Cell Biol 1966;30:23-38.

${ }^{13}$ Weibel ER. Stereological methods. Vol 2. London: Academic Press, 1980:151:

${ }^{14}$ Hicken P, Brewer D, Heath D. The relation between the weight of the right ventricle of the heart and the internal surface area and number of alveoli in the human lung in emphysema. $J$ Pathol Bacteriol 1966;92:529-46.

${ }^{15}$ Cleveland WS. Robust locally weighted regression and smoothing of scatterplots. Journal of the American Statistical Association 1979;74:829-36.

${ }^{16}$ Armitage P. Statistical methods in medical research. Oxford: Blackwell Scientific Publications, 1971;356.

${ }^{17}$ Weibel ER. Stereological Methods. Vol. 1. London: Academic Press, 1979:154.

Requests for reprints to: Professor J Swanson Beck, Pathology Department, Ninewells Hospital and Medical School, PO Box 120, Dundee DD1 9SY, Scotland. 